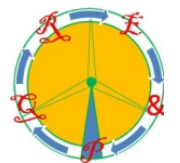

\title{
Design of Power Quality Virtual Lab Toolbox using LabVIEW/Multisim
}

\author{
S. Haidar ${ }^{1}$, E. Moussa ${ }^{1}$, M. El Hassan ${ }^{1}$, M. Badawi El Najjar ${ }^{1}$ \\ ${ }^{1-}$ Department of Electrical Engineering \\ University of Balamand \\ Kelhat - Al Kurah, (Lebanon) \\ Phone:+961 6 930250, e-mail: sandrahaidar@hotmail.com,moustapha.elhassan@balamand.edu.lb, \\ maged.najjar@balamand.edu.lb
}

\begin{abstract}
This paper presents a Power Quality (PQ) virtual lab that can be used by electrical engineers (EE) to enhance their knowledge and awareness on power quality disturbances in accordance to power quality standards. It will offer the EE the facility to become more aware about the problems tackling power systems and nonlinear devices, and their effects on the power quality indices. This work is built using NI LabVIEW/Multisim and is composed out of many simulations and experiments each with its learning objectives. The established measured power quality indices are mainly the root mean square (RMS), the total harmonic distortion (THD), the distortion index (DIN), the telephone influence factor (TIF), the crest factor (CF), the voltage transformer product (VT), the current transformer product (IT), the displacement power factor (DPF), the true power factor (TPF) and the unbalance factor (UF). Each of these indices is measured and analyzed in order to check how they are affected by the PQ issues.
\end{abstract}

Key words. Power quality virtual lab, voltage sag, voltage swell, harmonics, power quality indices

\section{Introduction}

Power quality, as defined by Kannan et al., is "a set of electrical boundaries that allows equipment to function in its intended manner without significant loss of performance or life expectancy." [1]

According to the International Conference on renewable and sustainable energy in 2013, power quality is a major concern in the electric power industry [1]. Its main goal is to offer the consumers a continuous sinusoidal voltage of a fixed amplitude [2]. It is an important issue in design performance for engineers, users, and workers. Unsuccessful operations are considered dangerous and will lead to economic losses in the industry which will certainly affect the production cycle.

All electrical devices in which power electronics and nonlinear loads are integrated, are considered sensitive to power quality disturbances [3] such as electric motors, computers, generators, transformers, or household appliances. Any issue that occurs in voltage, current or frequency variation may lead to a failure in the equipment, which is referred to as power quality problems [4].
Nowadays, economic losses have remarkably expanded due to the disturbances in power supply, and the major cause behind that is the universal usage of electronics in industrial and commercial installations. The connection between customers and companies has changed due to the liberalization of energy markets that makes power quality goals of an importance to all power system drivers.

In the recent years, the massive use of highly sensitive equipment is leading to more serious and significant economic losses. A "research on economic loss of power quality disturbances by econometric model" was done in 2010 concerning this topic. The results showed that in the US, 13.3 billion dollars are lost annually due to power quality disturbances [5]. Thus, quality loss and sensitivity of users are the two main features that can determine the severity of an economic loss caused by any disturbance in power systems.

As power quality problems are arising, the knowledge in power quality became a must for electrical engineers. It is important to help EE understand and experimentally quantify the effect of the power quality problems that can happen in both equipment and systems. As a result, EE will have enough knowledge to tackle and solve such a problem in accordance with the acquired power quality standards.

The work intends to form a virtual lab that points up all kinds of distortions, and their effects on PQ indices for a better learning process. This virtual tool presents itself as a feasible, eco-friendly and accessible learning tool as it doesn't require any equipment or electric components to use. Also, it doesn't require the usage of high voltages which will make it an efficient, risk-free and a fastlearning experience. Hence, this virtual lab is proposed to be a training tool that will help in learning and executing the concepts of power quality.

Throughout this paper, a detailed description for the methodology of the work will be discussed covering the main power quality disturbances such as voltage sag, voltage swell, interruption, harmonics and unbalance. In 
addition, many power quality indices affected by these PQ issues are tackled and elaborated in this paper including the RMS, THD, DIN, TIF, CF, VT, IT, DPF, TPF and UF measures. These power quality indices are crucial in representing, quantifying and comparing complex PQ phenomena. A graphical user interface will present all the results that will be analyzed with compliance to the power quality standards.

\section{Proposed System}

The designed virtual lab is accomplished using NI LabVIEW/Multisim, a user-friendly learning tool.

LabVIEW is an engineering workbench virtual tool used and considered as a graphical user interface software that possess a rich library and open development platform [6]. It is a graphical National Instrument product that uses block diagrams in designing instead of writing programming codes. It contains functions that are easily understood and utilized by the user, and a variety of features and tools that are familiar to EE [7]. This set of tools can be used for acquiring, analyzing, displaying and storing data [8].

The model consists of a three-phase source designed in LabVIEW, and of a three-phase RLC load built in Multisim and integrated in LabVIEW to form a closed circuit (Fig. 1). This will help EE to investigate the effects of power quality issues on different types of loads designed using Multisim. In addition, any circuit built using Multisim can be integrated in the PQ virtual lab to be analyzed in accordance with the PQ indices such as the circuit of an inverter or a DC motor.

Some measures of power quality indices were done directly through LabVIEW blocks, others were designed using numeric and mathematical blocks to execute the appropriate operations.

The implementation of these steps in LabVIEW will yield to an interface, and the integration of all the individual components will allow the formation of a virtual lab able to showcase the effect of disturbances on indices.

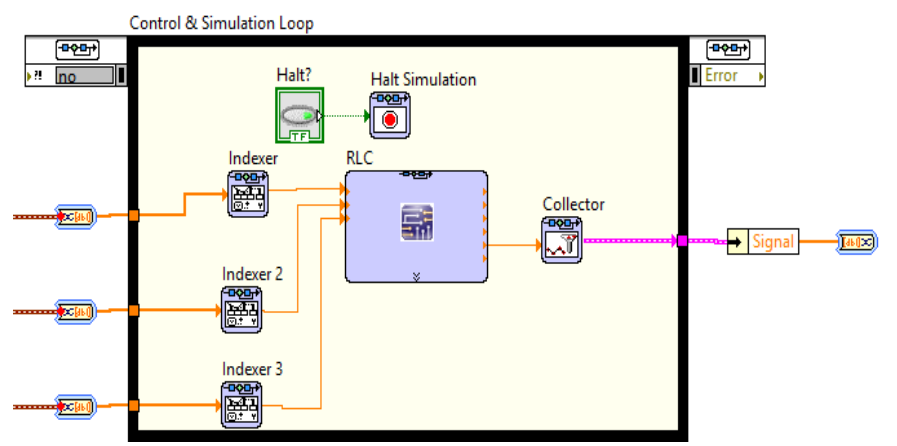

Fig. 1. Multisim Circuit in LABVIEW

Fig. 1 shows the model of Multisim integrated in LabVIEW. The parameters in the load RLC can be manipulated from Multisim. This load is connected to the three-phases source and its output is collected to be measured and analyzed.

\section{Power Quality Problems}

Power quality disturbances occurring in power systems and nonlinear devices are classified between short duration voltage deviations that are reasoned by fault conditions such as voltage sag, voltage swell, and interruptions, and between waveform distortion such as harmonics, and unbalance.

\section{A. Voltage Sag}

Voltage sag can be classified as a short duration voltage variation and is defined as a reduction for a short time in the RMS voltage for a value ranging from $10 \%$ to no more than $90 \%$ of the nominal voltage [4]. This voltage deviation can be caused by a phase fault in a power system, starting a large machine, or a step load [4].

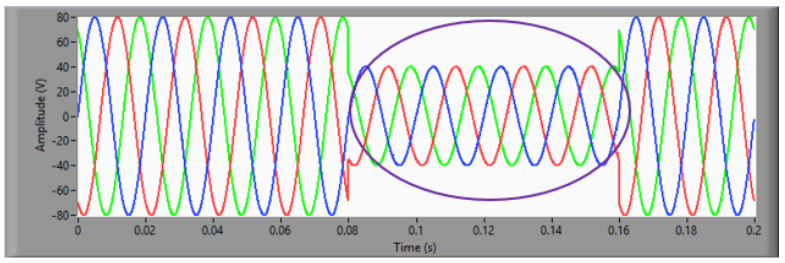

Fig. 2. Graph of the 3 Phase Voltage Vs. Time with a $50 \%$ Voltage Sag.

Fig. 2 shows the graph of a three-phase voltage with a sag at a given time. A clear decrease in the voltage is seen between $0.08 \mathrm{~s}$ and $0.16 \mathrm{~s}$. The voltage before $0.08 \mathrm{~s}$ and after $0.16 \mathrm{~s}$ is $80 \mathrm{~V}$, it decreases to $40 \mathrm{~V}$ between $0.08 \mathrm{~s}$ and $0.16 \mathrm{~s}$, then it goes back to $80 \mathrm{~V}$ after $0.16 \mathrm{~s}$. The decrease in the voltage is clearly defined as the occurrence of a $50 \%$ sag in the voltage. Therefore, a sag voltage is detected when the voltage decreases within a short duration of time for a value of no less than $10 \%$ of the nominal voltage.

\section{B. Voltage Swell}

In contrast to voltage sag, voltage swell is a rise in the RMS rate of the voltage for a value above $110 \%$ of the nominal RMS voltage [4]. It is caused by clearing a fault on a single phase of the three-phase system or by a sudden loss of a large load.

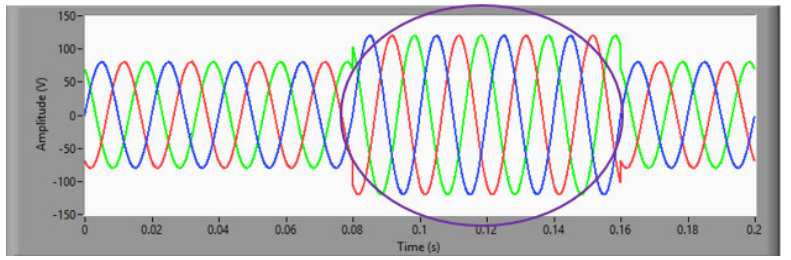

Fig. 3. Graph of the 3 Phase Voltage Vs. Time with a $50 \%$ Voltage Swell.

Fig. 3 shows the graph of a three-phase voltage with a swell in a specified period of time. It is seen that the voltages in the period varying from $0.08 \mathrm{~s}$ to $0.16 \mathrm{~s}$ increase to $120 \mathrm{~V}$ (shown above in the circle) which are greater than the voltages in the other periods ( 0 to $0.08 \mathrm{~s}$ and $0.16 \mathrm{~s}$ to 0.2 ) that have $80 \mathrm{~V}$ as their peak values. 


\section{Interruption}

The interruption is also considered a power quality disturbance. It occurs for a small period of time when the RMS voltage drops to less than 0.1 p.u or when the RMS voltage is completely lost. Interruptions are due to faults in power systems which may lead to a damage in the equipment [7]

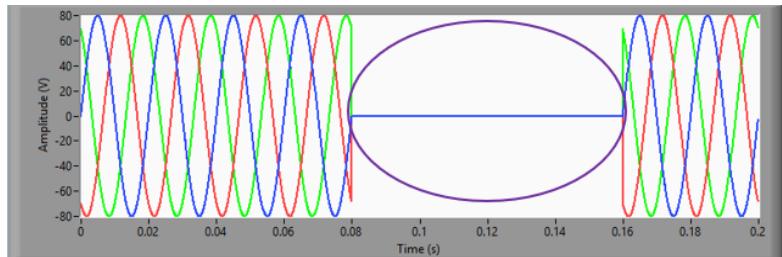

Fig. 4. Graph of the 3 Phase Voltage Vs. Time with a voltage interruption.

In Fig. 4, a complete loss of voltage is seen between $0.08 \mathrm{~s}$ and $0.16 \mathrm{~s}$ which indicates an interruption in the voltage source. Whereas before $0.08 \mathrm{~s}$ and after $0.16 \mathrm{~s}$ the signal is in its normal sinusoidal shape with the same amplitude $(80 \mathrm{~V})$.

\section{Harmonics}

Harmonics are present in sinusoidal voltages and currents and appear in all frequencies. However, in the industrial field, the frequencies range between 50 to $60 \mathrm{~Hz}$. The appearance of harmonics is due to the high usage of nonlinear devices and nonlinear loads in power systems [4]. These harmonics are defined as a sum of sine waves of different frequencies but multiples of the fundamental frequency. Therefore, a signal containing many harmonic components can be decomposed to more than one signal with different frequencies. A sinusoidal signal loses the proper shape of a sine wave when it becomes distorted with harmonics but remain a periodic signal.

Up to 50 harmonics can appear in a system. However, only three harmonics can be added in this virtual tool as its main purpose is to learn and investigate the basics and measures of power quality.

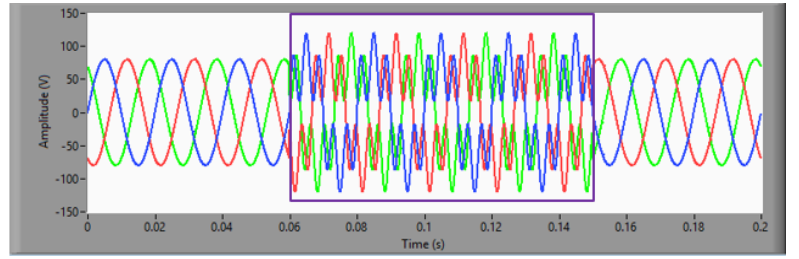

Fig. 5. Graph of the 3 Phase Voltage Vs. Time with the distortion of Harmonics of Order 3 And 5.

Fig. 5 shows a three-phase voltage distorted with two harmonic components of order 3 and 5 occurring between $0.06 \mathrm{~s}$ and $0.15 \mathrm{~s}$. Having the original shape of sinusoidal sinewave before $0.06 \mathrm{~s}$ and after $0.15 \mathrm{~s}$, the signal became distorted within this interval of time when the sinusoidal waves lose its normal shape.

\section{E. Unbalance}

Voltage unbalance occurs when the RMS magnitudes of the phases are different or when the phase angle between each two consecutive lines is different. Unbalance occurs when loads are added on one phase more than the other phases in a system [9].

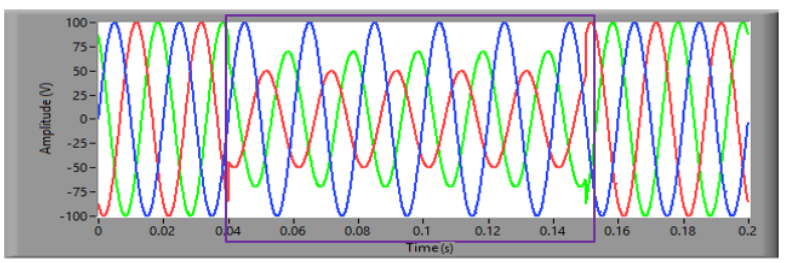

Fig. 6. Graph of the 3 Phase Unbalanced Voltage

An unbalance is shown in Fig. 6 between $0.04 \mathrm{~s}$ and $0.15 \mathrm{~s}$ where each phase has a different RMS magnitude from the others.

\section{User Interface}

As all the simulations are attached, all the equations and formulas are plugged in and designed in LabView, the EE can start creating the desired power quality issue and can start collecting the appropriate data.

The power quality virtual lab interface will pop up in front of the user to start the process experimenting many power quality disturbances. The EE can create a voltage sag, voltage swell, harmonics, interruption, unbalance and also a combination of more than one disturbance.

From the main Tab as shown in Fig. 7, the EE will set the voltage peak of the sine wave source, the frequency, and the duration of the signal in their corresponding control blocks. The fundamental waveform will appear in the graph below.

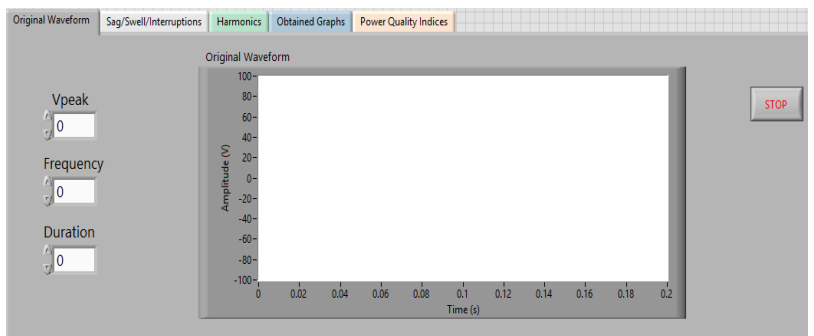

Fig. 7. Power Quality Virtual Lab Main Tab

In the next tab in Fig. 8, the user will choose whether the desired change is a sag, swell or interruption, then will set the magnitude of the sag or swell in form of percentage and the start and end time in their corresponding places. It is crucial to mention that the EE should pay attention in choosing the start and end time of the voltage disturbance within the duration of the signal to actually see the desired changes. For example, in Fig. 3 the magnitude of the sag voltage between $0.08 \mathrm{~s}$ and 0.16 s was about $50 \%$ of the nominal voltage. 


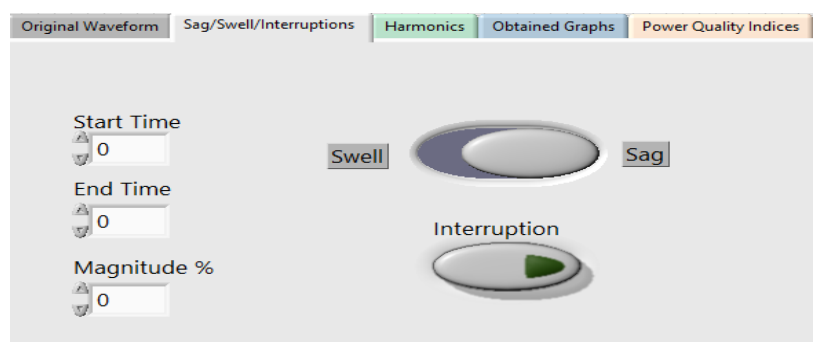

Fig. 8. Power Quality Virtual Lab- Voltage Deviations Tab

Not limited to that only, the user is able to add harmonics to the signal by moving to the next tab called "Harmonics" where the order and magnitude of each harmonic component and the duration of the distorted signal can be specified as shown in Fig. 9.

Here it is important to mention that the addition of harmonics in a signal can also be done by identifying the phase angle harmonic.

However, adding harmonics to the signal by defining the order of each harmonic along with its amplitude would be easier for learning and teaching purposes as the main goal is to investigate the basic concepts of PQ.

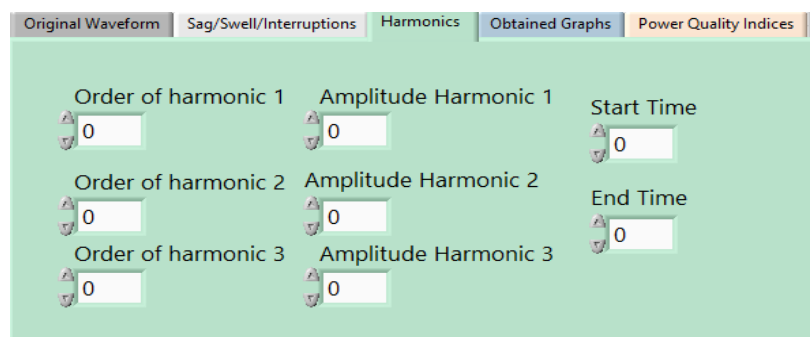

Fig. 9. Power Quality Virtual Lab - Harmonic Control Tab

As for the unbalance, it can be created in the Unbalance Control Tab as shown in Fig. 10, by either specifying the phase of unbalance voltages or by setting the peak voltage of each phase, and the desired frequency and duration of the signal.

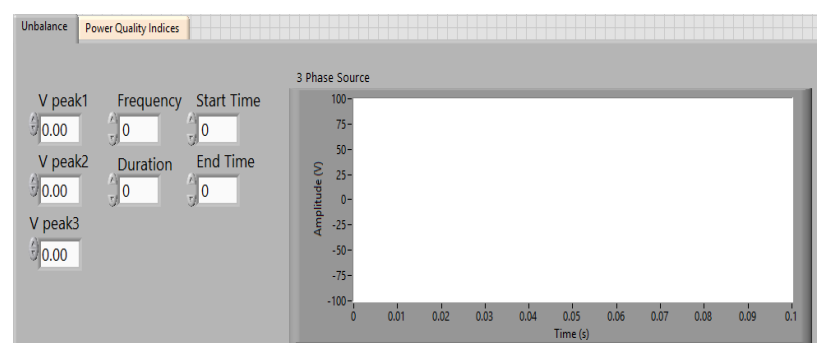

Fig. 10. Power Quality Virtual Lab - Unbalance Control Tab

The virtual tool allows the users to choose the type of disturbance they want to investigate, or a combination of disturbances of his choice. In addition, they are able to choose the start and end time of each disturbance separately.

Once these steps are accomplished and the model starts running, the results will appear in the next two Tabs termed respectively "Obtained graphs" and "Power quality indices".

\section{Simulation}

Many simulations and experiments were prepared to help the EE enhance their knowledge in power quality and become familiar with major power quality issues and indices. The plot results of the voltage and current along with the measured parameters will help the user to come out with the right analysis and interpretation on power quality in terms of indices and problems.

Example 1: Two types of disturbances at two different time were simulated using the virtual lab toolbox.

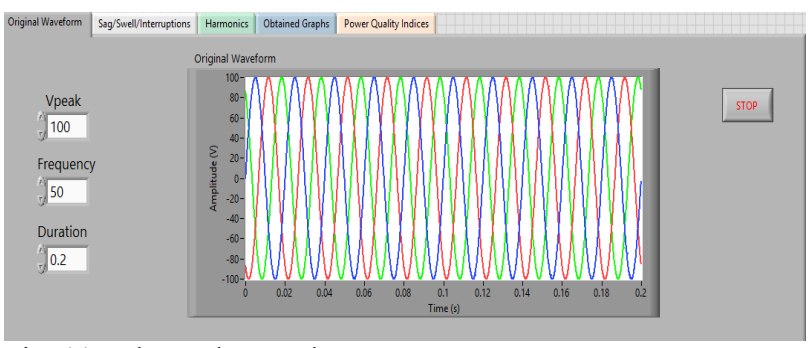

Fig. 11. Three-phase voltage source

Fig. 11 represents the original waveform where the values of the voltage peak, the frequency, and the duration of the signal were set by the user. This fundamental waveform is free from any disturbance.

As it is seen in Fig. 12, the push button is set on the sag side, therefore a sag voltage of $60 \%$ of the nominal voltage tackles the signal between $0.02 \mathrm{~s}$ and $0.06 \mathrm{~s}$.

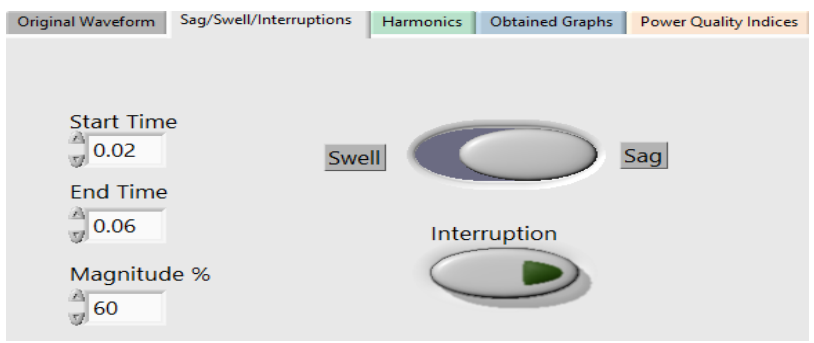

Fig. 12. Sag Voltage

In addition to the sag created, the signal is distorted by three harmonic components of order 3,5 and 7 with magnitudes of $10 \mathrm{~V}, 50 \mathrm{~V}$ and $75 \mathrm{~V}$ respectively, as shown in Fig. 13. These harmonics interfere in the signal between $0.06 \mathrm{~s}$ and $0.12 \mathrm{~s}$.

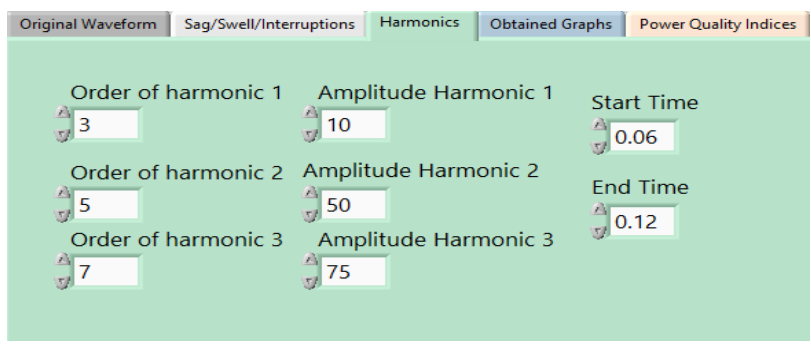

Fig. 13. Addition of Harmonics

The obtained results of the voltage and current signals are shown in Fig. 14 where plot (a) represents the threephase voltage source, plot (b) is the single-phase voltage, and plot (c) is the single-phase current. The two 
disturbances are clearly marked in the first plot of Fig. 14. The first rectangle represents a sag voltage that is less than the fundamental voltage. The second rectangle highlights the distorting signal consisting of the fundamental harmonic component along with the three added harmonics.

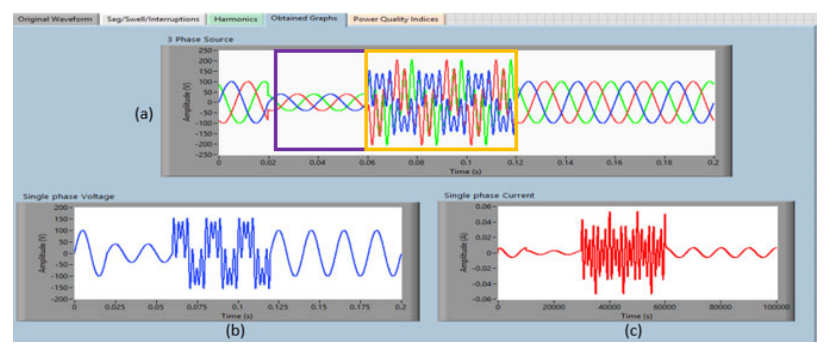

Fig. 14. Plot Results

Throughout this paper, the power quality indices are being measured and analyzed before, during, and after the occurrence of any disturbance.

Some of the PQ indices were generated directly through LabVIEW blocks, others were designed using mathematical blocks to perform the right operations.

These PQ indices are as follow:

\section{A. Total Harmonic Distortion (THD) \& Distortion Index (DIN)}

The total harmonic distortion (or THD) is a degree that displays the amount of voltage or current harmonic distortion. It is related to the amplitudes of the harmonics. As the distortion increases, the THD becomes indefinitely large.

$$
T H D_{v}=\frac{\sqrt{\sum_{i=2}^{\infty}\left(V_{r m s}{ }^{(i)}\right)^{2}}}{V_{r m s}{ }^{(1)}}
$$

On the other hand, a relation does exist between DIN and THD in the following equation:

$$
D I N_{I}^{2}=\frac{T H D_{I}^{2}}{1+T H D_{I}^{2}}
$$

Therefore, for low distortion, DIN is approximately equal to THD.

\section{B. Telephone Influence Factor (TIF)}

The TIF is equivalent to the THD taking into consideration its effect on the human ear. The harmonic magnitudes will be evaluated with factors called TIF weights. The value of these weights is a result of audio-logical and physiological tests on the human ear.

TIF weights $w_{i}$ are factors that depend on the frequency initially used in the system where each order of harmonic has its own TIF weight factor. These factors will be multiplied by the magnitude of each harmonic in order to compute TIF. It is expressed by the following equation.

$$
T I F_{v}=\frac{\sqrt{\sum_{i=1}^{\infty}\left(w_{i} V_{R M S}{ }^{(i)}\right)^{2}}}{\sqrt{\sum_{i=1}^{\infty}\left(V_{R M S}{ }^{(i)}\right)^{2}}}
$$

\section{Distortion Power Factor}

The distortion power factor is caused by the current harmonic distortion. It is a measure of how much this harmonic distortion affects the power transferred to the load. It is related to the $T H D_{I}$ by the following formula:

$$
\text { DistortionFactor }=\frac{1}{\sqrt{1+T H D_{I}^{2}}}
$$

\section{Displacement Power Factor DPF and True Power Factor (TPF)}

The displacement power factor is an index that measures the phase shift between the fundamental voltage and current components. Whereas, the true power factor represents how efficient and effective a system is when converting the input voltage and current into active power.

$$
\begin{gathered}
D P F^{3}=\left|\cos \left(\varphi_{f_{0}}\right)\right| \\
T P F=\frac{P}{S}=D P F \times \text { DistortionFactor }
\end{gathered}
$$

\section{E. Voltage Transformer Product (VT)}

The VT product takes into account the strength of the signal and represents the audio interference resulting from the Bus voltages. It is equal to the TIF weights multiplied by the magnitude of harmonic components.

$$
T I F_{v} \times V_{r m s}=V T
$$

\section{F. Crest Factor}

The crest factor is known as peak values. It estimates the amount of transient phenomena happening in a signal.

$$
C F=\frac{V_{p e a k}}{V_{r m s}}
$$

\section{G. Unbalance Factor}

The unbalance factor is a measure of how much the phases of the system are unbalanced. It is explained as the quotient of the modulus of the negative sequence to the positive sequence of the voltage.

$$
U F=\frac{\left|V^{-}\right|}{\left|V^{+}\right|}
$$


The computation of the PQ indices on the implemented system along with the RLC load is identified before any disturbance, during the sag voltage, during the harmonic distortion and after these disturbances. These measures are displayed in the table below (Table 1) to clearly analyze how the parameters are affected by each disturbance.

Table I. Comparison Table of the values of power quality indices

\begin{tabular}{l|l|l|l|l|} 
& $\begin{array}{l}\text { Before } \\
\text { Disturbances }\end{array}$ & During Sag & $\begin{array}{l}\text { During } \\
\text { Harmonic }\end{array}$ & $\begin{array}{l}\text { After } \\
\text { Disturbances }\end{array}$ \\
\hline Vrms (V) & 70.5346 & 28.2843 & 95.38 & 70.71 \\
\hline THDv (\%) & 0 & 0 & 90.69 & 0 \\
\hline TIFv & 0 & 0 & 237.607 & 0 \\
\hline VT & 0 & 0 & 22662.9 & 0 \\
\hline $\begin{array}{l}\text { Distortion } \\
\text { Factor }\end{array}$ & 1 & 1 & 0.183211 & 1 \\
\hline $\begin{array}{l}\text { Displacement } \\
\text { Power Factor }\end{array}$ & 0.066284 & 0.06572 & 0.06567 & 0.06626 \\
\hline $\begin{array}{l}\text { True Power } \\
\text { Factor }\end{array}$ & 0.066284 & 0.06572 & 0.01203 & 0.06626 \\
\hline
\end{tabular}

Table I indicates that the RMS value is affected by all types of disturbances. When no disturbance occurs, the RMS voltage is almost $70.7 \mathrm{~V}(V p \sqrt{2}=100 \sqrt{2}=70.7)$. This RMS value decreases in the case of a sag voltage (28.28 $\mathrm{V})$ and increases in the case of a swell voltage or harmonic distortion $(95.38 \mathrm{~V})$.

The crest factor, in a normal signal, free of harmonics, is equal to almost 1.414 and this index varies with the variation of the RMS and peak voltages.

However, THD, DIN, TIF, VT, and IT are only affected in the presence of harmonics. Otherwise, they are equal to zero. The harmonic plot in a normal signal only contains the fundamental component. Whereas, the harmonic plot of a distorted signal shows the fundamental component along with the $3 \mathrm{rd}, 5$ th and 7 th order harmonics. In this case, the THD, DIN, TIF, VT and IT will highly increase.

The distortion power factor related to the total harmonic distortion is equal to one in a signal free of harmonics. Hence, this factor decreases to 0.18321 during the presence of harmonic distortion.

While the displacement power factor remains the same even with the presence of harmonics, the true power factor gets smaller as harmonics are added and becomes less than the displacement factor. Both TPF and DPF are equal in non-harmonic situations.

\section{Conclusion}

In their field work, electrical engineers might face a lot of problems while dealing with power quality. All new technologies related to nonlinear devices and renewable energy sources generate a huge amount of quality disturbances.

This virtual lab was designed to enhance the knowledge of the EE on power quality problems, for them to be aware of all the issues and to be able to handle and manage all the situations properly in their future career as electrical engineers with regards to power quality standards and practice guides. Each PQ problem such as voltage sag, voltage swell, harmonics and unbalance were tackled in this virtual lab by measuring and analyzing all the PQ indices discussed earlier in the paper.

This tool is considered as a teaching and training tool, to be applied in lab activities after teaching, learning and discussing the power quality generalities and concepts. A laboratory manual with several experiments was developed to meet the learning objectives related to power quality.

This lab was built using NI LabVIEW/Multisim, an accessible learning tool. The EE will be able to investigate the effects of the desired power quality issue on different types of loads designed using Multisim.

Moreover, it is important to mention that this ecofriendly and cost-efficient virtual lab can be considered as a foundation because it can always be updated and developed for future objectives where more issues can be tackled and more experiments can be added to always keep EE fully informed on all the updates related to power quality.

\section{Acknowledgement}

The authors acknowledge the University of Balamand and especially the supervisors for their guidance, help, expertise, and support during the project.

\section{References}

[1] A. Kannan, V. Kumar, T. Chandrasekar and B. J. Rabi, "A review of power quality standards, electrical software tools, issues and solutions." 2013 International Conference on Renewable Energy and Sustainable Energy (ICRESE), Coimbatore, 2013, pp. 91-97.

[2] A. Tekale, D. Nawani, N. Belkar and V. Devkar, "A review paper on power quality issues and mitigation strategies," IAETSD Journal for Advanced Research in Applied Sciences, vol. 4, p. 2394-8442,2017

[3] IEEE Recommended Practice for Powering and Grounding Sensitive Electronic Equipment," in IEEE Std 1100-1992, vol., no., pp.1-256, 31 Dec. 1992

[4] V. Kavitha and K. Subramanian, "Investigation of power quality issues and its solution for distributed power system." 2017 International Conference on Circuit .Power and Computing Technologies (ICCPCT), Kollam, 2017, pp. 1-6.

[5] Tianyou Li, Huiru Zhao, Chunjie Li, Dachang Ou and Liwen Fu, "The research on economic loss of power quality disturbances by econometric model," CICED 2010 Proceedings, Nanjing, 2010, pp. 1-8.

[6] Keerthana Suganthi V., Madhuridevi L. and Sindhuja K., "Development of software tool for classroom teaching of power quality using LabVIEW," 2014 International Conference on Advances in Electrical Engineering (ICAEE), Vellore, 2014, pp. 1-6.

[7] LABVIEW, National Instruments. [Online]. Available: www.ni.com/labview/

[8] Y. Huping and B. Zhipeng, "The Power Quality Monitoring System Based on Virtual Instrument," 2009 WRI World Congress on Software Engineering, Xiamen, 2009, pp. 243-245.

[9] D. Bellan, "Statistical properties of voltage unbalance factor in three-phase power systems," International Journal of Applied Engineering Research, vol. 11, no. 15, p. 84758479,2016 